\title{
Monitoring of the atmospheric electric field and cosmic-ray flux for the inter- pretation of results in high-energy astroparticle physics experiments.
}

\author{
Ashot Chilingarian ${ }^{1, *}$, Johannes Knapp ${ }^{2}$, and Mary Zazyan ${ }^{1}$ \\ ${ }^{1}$ A. Alikhanyan National Lab (Yerevan Physics Institute), Yerevan, Armenia. \\ ${ }^{2}$ Deutsches Elektronen-Synchrotron, Zeuthen, Germany.
}

\begin{abstract}
Atmospheric electric fields influence experiments using the atmosphere as a detector for very weak fluxes of highest-energy gamma rays and protons/nuclei coming from galactic and extragalactic sources. Multiplication of electrons and gamma rays in strong atmospheric electric fields change particle numbers and energy spectra of the secondary shower particles and consequently influence the reconstructed properties of the primary particles. Here, we present a MC study using the CORSIKA package to explore and quantify these effects.
\end{abstract}

\section{Introduction}

Atmospheric electricity is always present in the atmosphere, enabling natural acceleration of charged particles. Potential differences in or nearby thunderclouds and between the Earth's surface and clouds accelerate everpresent free charged particles from interactions of gamma rays and cosmic rays with atoms of the atmosphere. The direction of the acceleration depends on the charge of particles and the field strength and orientation. The most prominent effects of strong atmospheric electricity are thunderstorm ground enhancements (TGEs) - huge fluxes of gamma rays, electrons and neutrons which can be detected on Earth's surface. On Mount Aragats in Armenia, the rather stable flux of secondary particles from cosmicray air showers are often multiplied many times for a several minutes in the presence of strong electric fields (see [3], [4], [5]), and the energy spectra of secondary particles can be changed significantly. Recently, even long-lasting TGEs of several hours duration have been discovered [8]. Thus, electric fields and processes in the atmosphere can have important consequences on experiments which measure very-high-energy gamma rays and cosmic rays from galactic and extragalactic sources. At high energies, such experiments use large volumes of atmosphere as an "amplifier" that transforms each of the rare primary particles into a particle shower of numerous lower-energy electrons, gamma rays, mesons, protons and neutrons, which is much easier to detect than the primary particles alone. Veryhigh-energy gamma rays, are detected with so-called atmospheric Cherenkov telescopes (ACTs) with effective areas of $10^{4}-10^{6} \mathrm{~m}^{2}$. Cosmic rays are detected with gigantic arrays of particle detectors on the Earth's surface, which register the secondary particles of extensive air showers (EAS) on up to $3 \times 10^{9} \mathrm{~m}^{2}$. In all air-shower experiments, the number of secondary particles in a shower is the basis

*e-mail: chili@aragats.am for the reconstruction of the energy of the primary particle and the longitudinal shower development is the key for the determination of the primary particle type. We discussed that these observables are susceptible to the atmospheric electric fields, and biases can occur not only during thunderstorms, but also several hours after storm ceased, due to the permanent presence of weak electric fields in the post-storm atmosphere. Crucial to the understanding of possible biases of the EAS parameters is the knowledge of the atmospheric electric fields. As was stated in [18], the intensity of EAS are radically changed after propagation in the atmospheric electric fields. To estimate their possible influence, we performed an initial Monte Carlo study with the CORSIKA code which shows that atmospheric electric fields should be considered in EAS physics.

\section{The electrical atmosphere and particle fluxes}

We use a new observable in the atmospheric electricity research, namely, the steady particle fluxes from the clouds to ground. The origin of the fluxes of electrons, gamma rays and neutrons detected on the Earth's surface are Runaway Breakdown (RB) processes [12], nowadays mostly referred to as Relativistic Runaway Electron Avalanches (RREA, [19], [10]) and MOdification of the energy Spectra of the electrons (MOS, [6]). Simultaneously to the measurements of cosmic-ray particles, also near-surface electrostatic fields and lightning strikes are recorded. The combination of detailed measurements of particle fluxes and spectra at ground and electric fields and lightning flashes, first investigated on Aragats, allows monitoring the formation of charge accumulations in the overhead atmosphere. The electron acceleration towards Earth is due to the electric field between the main negative charge region in the middle of the cloud and the positive charge that is induced by it on the ground. This field is 


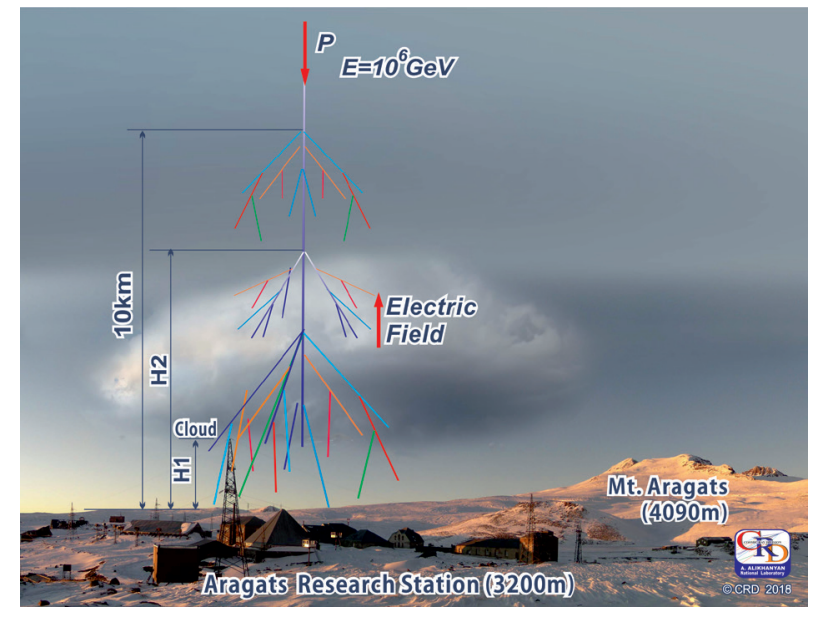

Figure 1. EAS development in an atmosphere with electric field.

significantly increased by the electric field between the main negative region and an emerging Lower Positive Charge Region (LPCR) still within the cloud. The maximal intensity (and maximal energy of particles) of TGEs is observed when the strength of the local electric field in the cloud exceeds the "runaway" threshold and RB/RREA avalanches start to develop downwards. Such conditions are met above the LPCR. The lightning leader cannot make its path through the LPCR, and cloud-to-ground flashes are suppressed [15].

The gamma-ray flux attenuates due to lightning flash that brings free electrons from the main negatively charged region to LPCR (inverted intracloud flash) or to the ground (cloud to ground flash). Thus, the charge in the layers, and consequently the electric field decreases and RB/RREA cascades vanish (see Figure 5 of [8]). However, the electric field in the cloud still remain disturbed and low energy gamma-ray flux continue.

In the cartoon (Figure 1) we show the electron-gamma ray avalanche developing in the bottom of the thundercloud above the Aragats high-altitude research station of the Yerevan Physics Institute [2]. The avalanche comes out of the base of the cloud and illuminates various particle detectors measuring count rates of charged and neutral particles and their energies. The distance to the cloud base in spring and autumn is rather short $\mathrm{H} 1=25-50 \mathrm{~m}$; in Summer, it is $\mathrm{H} 1=50-500 \mathrm{~m}$. In our simulation studies of TGEs we assume the strength of electric field in the cloud up to $1.8 \mathrm{kV} / \mathrm{m}$ extended over up to $\mathrm{H} 2-\mathrm{H} 1=1 \mathrm{~km}$. Values of electric field and elongation used in simulation have been measured in balloon flights [14].

In the Figure 2, we show two long-lasting TGEs occurred on August 17 and October 10, 2017. The particle flux remained higher for $\sim 1.5$ hours after the decline of the thunderstorm. Electric field sensors, so called electric mills, located near the particle detectors do not record any significant disturbances and only by the particle detectors counts, we can deduce that there is still a small electric field in the atmosphere that can affect characteristics of air show-

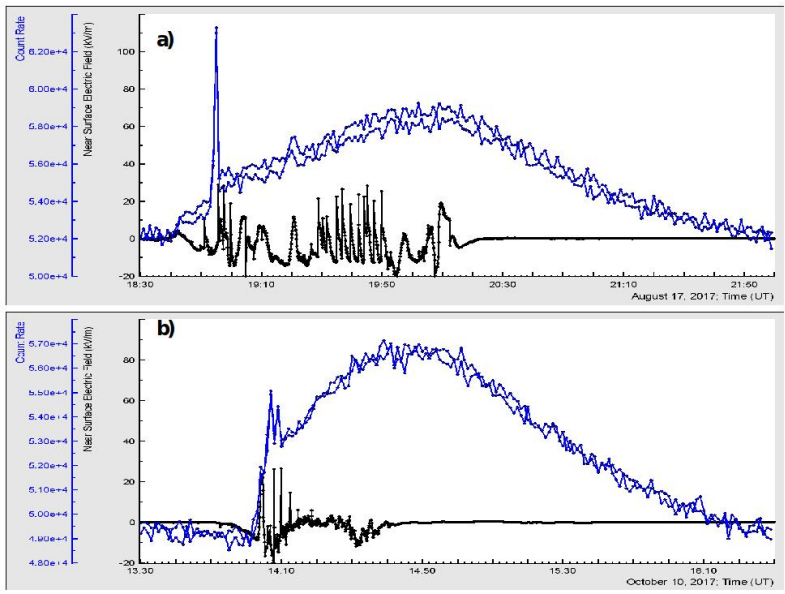

Figure 2. Near-surface electric field (black) and count rates (blue) of NaI detectors with energy threshold of $300 \mathrm{keV}$, for two long-lasting, low-energy TGEs.

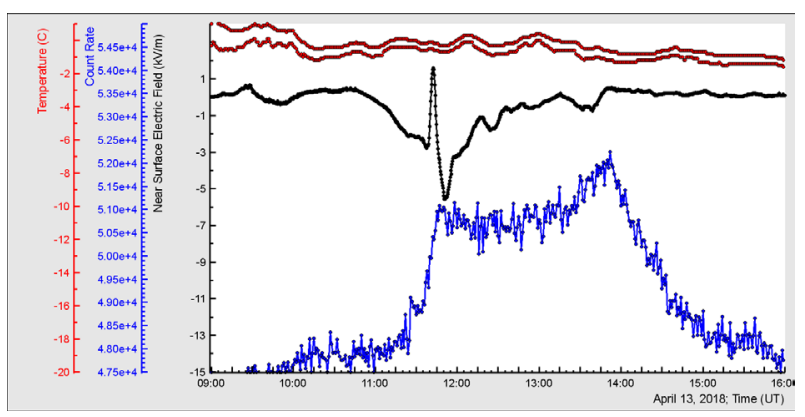

Figure 3. Red:outside temperature and dew point; black: nearsurface electric field in $\mathrm{kV} / \mathrm{m}$; blue: one-minute time series of the count rate in a $1 \mathrm{~m}^{2}$ plastic scintillator $(1 \mathrm{~cm}$ thick, energy threshold $0.7 \mathrm{MeV}$ ).

ers (number of electrons and gamma rays reaching Earth's surface). Thus, the monitoring of the near-surface electric field at the detector site is necessary, but it is not a sufficient condition for evaluating the intracloud electric field. A simple NaI crystal based spectrometer that monitors particle flux on one second time scale and stores histogram of energy released each minute will not only provide data for correction of EAS parameters, but will also give valuable data on one of the most complicated and most important topics of atmospheric physics: the atmospheric electricity. By the intensity and energy spectrum of TGE, it is possible to roughly estimate the potential drop in the cloud [20], [9]. Electric field effects of 1-2 hours' duration are not the limit for the electrically disturbed atmosphere. In Figure 3 we show enhanced particle fluxes prolonged for $\sim 6$ hours, thus demonstrating prolonged electrical activity in clouds above detector site. Estimated distances to cloud base do not exceed $50 \mathrm{~m}$. Note that the variations of the near-surface electric field were rather small from -5 to $1 \mathrm{kV} / \mathrm{m}$. In Figure 1, disturbances were $-20 \mathrm{kV} / \mathrm{m}$ to $10 \mathrm{kV} / \mathrm{m}$. Thus, a small near-surface electric field corresponds to low energy prolonged gamma-ray fluxes. 
Table 1. Average number of shower particles measured on ground per $10^{15} \mathrm{eV}$ vertical proton shower.

\begin{tabular}{|c|c|c|c|c|}
\hline & $\begin{array}{l}E_{z}=0 \\
\mathrm{kV} / \mathrm{cm}\end{array}$ & $\begin{array}{c}E_{z}=1.8 \\
\mathrm{kV} / \mathrm{cm} \text { at } \\
\mathrm{H}>3250 \mathrm{~m} \\
\mathrm{H}<4250 \mathrm{~m}\end{array}$ & $\begin{array}{c}E_{z}=1.8 \\
\mathrm{kV} / \mathrm{cm} \text { at } \\
\mathrm{H}>4200 \mathrm{~m} \\
\mathrm{H}<5200 \mathrm{~m}\end{array}$ & $\begin{array}{c}E_{z}=1.0 \\
\mathrm{kV} / \mathrm{cm} \text { at } \\
\mathrm{H}>4200 \mathrm{~m} \\
\mathrm{H}<5200 \mathrm{~m}\end{array}$ \\
\hline$N_{e}$ & $4.03 \cdot 10^{5}$ & $7.25 \cdot 10^{5}$ & $4.34 \cdot 10^{5}$ & $4.08 \cdot 10^{5}$ \\
\hline$N_{\mu}$ & $11.48 \cdot 10^{3}$ & $11.73 \cdot 10^{3}$ & $12.09 \cdot 10^{3}$ & $11.70 \cdot 10^{3}$ \\
\hline$N_{\gamma}$ & $1.91 \cdot 10^{6}$ & $2.86 \cdot 10^{6}$ & $2.26 \cdot 10^{6}$ & $1.95 \cdot 10^{6}$ \\
\hline
\end{tabular}

Table 2. Relative changes of shower particles numbers (compered to the $E_{z}=0$ case).

\begin{tabular}{|c|c|c|c|}
\hline & $\begin{array}{c}E_{z}=1.8 \\
\mathrm{kV} / \mathrm{cm} \text { at } \\
\mathrm{H}>3250 \mathrm{~m} \\
\mathrm{H}<4250 \mathrm{~m}\end{array}$ & $\begin{array}{c}E_{z}=1.8 \\
\mathrm{kV} / \mathrm{cm} \text { at } \\
\mathrm{H}>4200 \mathrm{~m} \\
\mathrm{H}<5200 \mathrm{~m}\end{array}$ & $\begin{array}{c}E_{z}=1.0 \\
\mathrm{kV} / \mathrm{cm} \text { at } \\
\mathrm{H}>4200 \mathrm{~m} \\
\mathrm{H}<5200 \mathrm{~m}\end{array}$ \\
\hline$\Delta N_{e} / N_{e}$ & 0.80 & 0.08 & 0.01 \\
\hline$\Delta N_{\mu} / N_{\mu}$ & 0.02 & 0.05 & 0.02 \\
\hline$\Delta N_{\gamma} / N_{\gamma}$ & 0.50 & 0.18 & 0.02 \\
\hline
\end{tabular}

\section{Simulation of air showers with electric fields in the atmosphere using the CORSIKA code}

To study the influence of atmospheric electric fields on Extensive Air Showers (EAS) parameters, we developed a simple model for the propagation of shower particles in the atmosphere taking into account electric fields. Air showers have been simulated with CORSIKA [13] version 7.56 with QGSJETII [17] and GHEISHA [11] as hadronic interaction models. For electromagnetic interactions, the EGS4 option [16] was used. The electric field option (EFIELD) of CORSIKA was used. An electric field of strength $E_{z} \neq 0$ starts at a height $\mathrm{H} 2$ above the ground and extends to height H1. Each simulation trial consists of propagation of hundred of vertical proton showers with fixed primary energy of $10^{15} \mathrm{eV}$. The showers start on the top of atmosphere (first interactions are most probable on heights of $30 \mathrm{~km}$ ), the secondary particles are propagated and recorded at the altitude of $3200 \mathrm{~m}$ (Mt. Aragats station). Shower particles are followed down to $0.3 \mathrm{GeV}$ for hadrons and muons, and to $0.3 \mathrm{MeV}$ for electrons and gamma rays. The simulations show a significant influence of the electric field on the intensity of particles at the ground level. In Table 1, we show the multiplication of the shower particles entering electric fields of different strength and location in the atmosphere. The particles reaching the Earth's surface are registered if their energy exceeds $50 \mathrm{keV}$. Thus, if the electric field extends down to only $50 \mathrm{~m}$ above ground (a common condition for the Spring TGEs on Aragats), the multiplication of electrons and gamma rays is rather significant. In Table 2, the relative changes in particle number are presented. The changes in the number of charged particles of an air shower depend on the strength of the field and on the height of the electric field. Figures 4 and 5 illustrate the effect of electric field on particle intensity at the ground level. Thus, we see that the electric field located

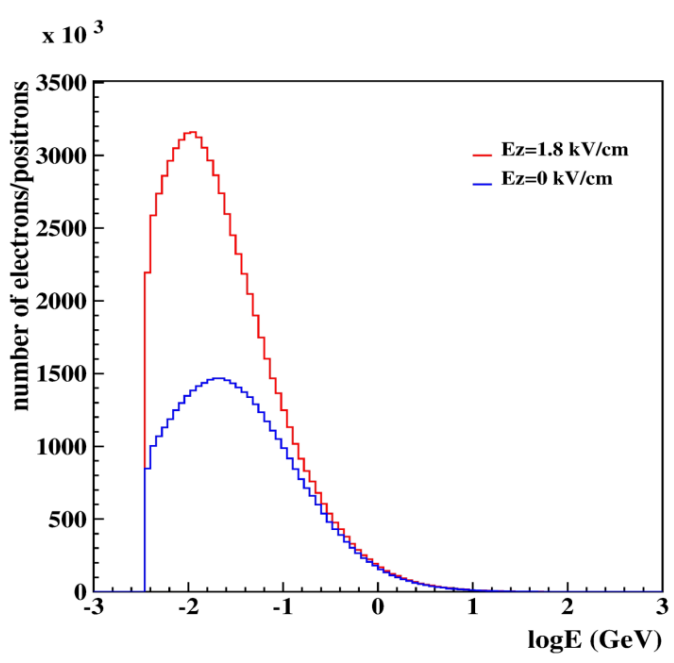

Figure 4. Energy distributions for the secondary electrons/positrons.

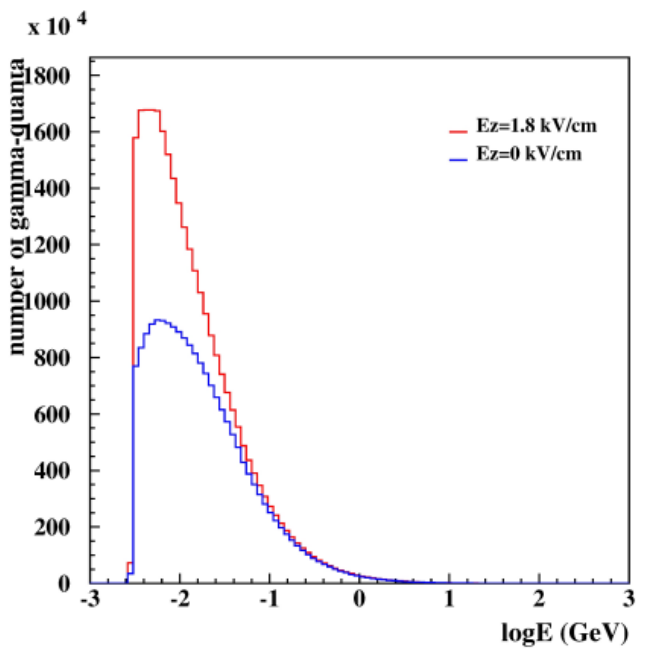

Figure 5. Energy distributions of secondary gamma rays in a shower, recorded at the ground level.

in the Earth's atmosphere drastically changes the number of charged and neutral particles of an air shower. This effect is essential for the estimation of EAS primary particle energy. Usually the energy of primary particle is estimated by the number of electrons and muons observed on ground level. For the CASA-MIA detector [21] (energies $10^{14} \mathrm{eV}-10^{16} \mathrm{eV}$ ) a combination of measured EAS parameters $\left(\mathrm{N}_{e_{*}}+25 \mathrm{~N}_{\mu}\right)$ has been found [22] to be logarithmically linear with energy (the subscript " $\mathrm{e}$ *" emphasizes that the quantity Ne does not simply denote the total number of electrons at the ground, but also includes a fraction of the abundant shower photons). For comparison, we use the parametrization:

$$
\log E_{0}[G e V]=1.03 * \log \left(N_{e}+25 N_{\mu}\right)
$$

Here, $N_{e}$ is the number of electrons and positrons, and the coefficient 1.03 takes also gamma rays contamination into 
Table 3. Average number of shower particles measured on ground per $10^{15} \mathrm{eV}$ vertical proton shower.

\begin{tabular}{|c|c|c|c|c|}
\hline & $E_{z}=0$ & $E_{z}=1.8$ & $E_{z}=1.8$ & $E_{z}=1.0$ \\
& $\mathrm{kV} / \mathrm{cm}$ & $\mathrm{kV} / \mathrm{cm}$ at \\
& & $\begin{array}{c}\mathrm{HV}>3250 \mathrm{~m} / \mathrm{cm} \text { at } \\
\mathrm{H}<4250 \mathrm{~m}\end{array}$ & $\begin{array}{c}\mathrm{H}>4200 \mathrm{~m} / \mathrm{cm} \text { at } \\
\mathrm{H}<5200 \mathrm{~m}\end{array}$ & $\begin{array}{c}\mathrm{H}>4200 \mathrm{~m} \\
\mathrm{H}<5200 \mathrm{~m}\end{array}$ \\
\hline $\mathbf{E}(\mathrm{GeV})$ & $10^{6}$ & $1.54 \cdot 10^{6}$ & $1.1 \cdot 10^{6}$ & $1.05 \cdot 10^{6}$ \\
\hline
\end{tabular}

account. The true and recovered energies according to eq. 1 are presented in Table 3.

With a field strength of $\mathrm{E}_{z}=1.8 \mathrm{kV} / \mathrm{cm}$, the errors of the primary particle energy reconstruction are $54 \%$ (electric field at $3250 \mathrm{~m}<\mathrm{H}<4250 \mathrm{~m}$, i.e. the cloud just 50 above ground); and $10 \%$ (electric field at $4200 \mathrm{~m}<\mathrm{H}<5200$ $\mathrm{m})$. With a field strength of $E_{z}=1.0 \mathrm{kV} / \mathrm{cm}$, the bias of the primary particle energy reconstruction is $5 \%$ (electric field at $4200 \mathrm{~m}<\mathrm{H}<5200 \mathrm{~m}$ ).

\section{Conclusion}

To study the influence of atmospheric electric fields on EAS parameters, we use a simple model of the propagation of shower particles initiated from a primary proton with energy of $1000 \mathrm{TeV}$. Observing particle fluxes from thunderclouds with electrical fields inside helps to estimate the influence of atmospheric electricity on the size of shower particles. The recent discovery of long-lasting TGEs extends the expected time of atmospheric electric field effects to several hours; thus, the influence of atmospheric electricity on the secondary cosmic-ray particles is potentially a long-lasting phenomenon. MC results prove that atmospheric electric fields should be considered in EAS physics.

\section{Acknowledgment}

The authors thank the staff of the Aragats Space Environmental Center for the continuous operation of Aragats research station facilities. The data for this paper are available via the multivariate visualization software ADEI on the WEB page of the Cosmic Ray Division (CRD) of the Yerevan Physics Institute, http://adei.crd.yerphi.am/adei.

\section{References}

[1] Babich L.P., Donskoy E.N., Il'kaev R.I., Kutsyk I.M., and Roussel-Dupre R.A., Plasma Phys. Rep. 30, 616 (2004).
[2] Chilingarian, A., Arakelyan, K., Avakyan, K. et al., Nucl. Instrum. Methods A 543 (2-3) 483 (2005).

[3] Chilingarian, A., Daryan A., Arakelyan K. et. Al., Phys. Rev. D, 82, 043009 (2010).

[4] Chilingarian, A., Hovsepyan, G., Hovhannisyan, A., Phys. Rev. D: Part. Fields, 83 (6), 062001 (2011).

[5] Chilingarian A., Journal of Atmospheric and SolarTerrestrial Physics, 107, 68-76 (2014).

[6] Chilingarian A., Mailyan B. and Vanyan L., Atmospheric Research 1, 114-115 (2012).

[7] Chilingarian A., Hovsepyan G., and Vanyan L., Europhys. Lett., 106, 59001 (2014).

[8] Chilingarian A., Physical Review D, 98, 022007 (2018).

[9] Cramer E.S., Mailyan B. G., Celestin S.,and Dwyer J. R., J. Geophys. Res. Atmos., 122, 4763-4772 (2017).

[10] Dwyer J.R, Geophys. Res. Lett., 30, 2055 (2003).

[11] Fesefeldt H., (1985), The simulation of hadronic showers - physics and applications, Tech. Rep.PITHA85/02, RWTH Aachen.

[12] Gurevich A.V., Milikh G.M. and Rouseel-Dupre R., Physics Letters A, 165, 463-468 (1992).

[13] Heck D., Knapp J., et al., CORSIKA: A Monte Carlo Code to Simulate Extensive Air Showers, Report FZKA 6019 (1998), Forschungszentrum Karlsruhe.

[14] Marshall T.C., Stolzenburg M., Krehbiel P.R. et. al., JGR, 114, D02209 (2009).

[15] Nag A. and Rakov V.A., Geophys. Res. Lett., 36, L05815 (2009).

[16] Nelson W., Hirayama H., Rogers W., (1985), The EGS4 Code System, Tech. Rep. 265, SLAC.

[17] Ostapchenko S., arXiv:hep-ph/0501093 (2005).

[18] P.Schellart, T. N. G.Trinh, S.Buitink, et al., Physical Review Letters, 114, 165001 (2015).

[19] Babich, L.P., et al., IEEE Trans. Plasma Sci., 29 (3), 430-438 (2001).

[20] Chilingarian A., Hovsepyan G., Khanikyanc Y., Reymers A. and Soghomonyan S., EPL, 110, 49001 (2015).

[21] A. Borione et al., Nucl. Inst. Meth. Phys. Res. A, 346, 329 (1994).

[22] Glasmacher, M. A. K., et al., in Proc. 26th Int. Cosmic Ray Conf. (Salt Lake City), 3, 129 (1999). 